\title{
Application of analytic hierarchy process on the study of soil salinization in the Northern Yinchuan Plain, China
}

\author{
Chenchen Huo ${ }^{1,2, a}$, Yanyan Gao ${ }^{1,2, b}$, Hui Qian ${ }^{1,2, ~ c}$, Jia Yang 1, 2, d \\ ${ }^{1}$ School of Environmental Science and Engineering, Chang'an University, Xi'an 710054, China \\ ${ }^{2}$ Key Laboratory of Subsurface Hydrology and Ecological Effect in Arid Region of Ministry of \\ Education, Xi'an 710054, China \\ ahuochenchen91@163.com, bgaoyanyan6366@163.com, 'qianhui@chd.edu.cn, \\ dyangjiaat@163.com
}

Keywords: Soil salinization, the analytic hierarchy process (AHP), causes of soil salinization, prevention measures.

\begin{abstract}
The Northern Yinchuan Plain is a productive agricultural area of northwest China, which is under threat due to soil salinization. According to the formation and restoring history of soil salinization, the analytic hierarchy process (AHP) was adopted for analyzing the causes of soil salinization and efficiency of various restoring measures. The calculated results of the relative weights of the indicators showed that semi-arid climate and parent material of soil are dominant factors of soil salinization, which account for $40.1 \%$ and $21.4 \%$ respectively. Furthermore, afforestation and planting salt-tolerant plants and improving drainage system were better than other measures, the values were $38.4 \%$ and $29.9 \%$ respectively. The study showed that AHP method could provide meaningful references for preventing and improving soil salinization in the study area.
\end{abstract}

\section{Introduction}

Soil salinization of irrigated lands is a crucial worldwide environmental problem and has negatively influence on the development of sustainable agriculture, especially in arid and semi-arid regions [1]. The Northern Yinchuan Plain is a significant food production base located in the Ningxia, Northwest China. However, soil salinization is one of the most significant problems that affect the agricultural production of the area, which has been restricting the Northern Yinchuan Plain in social and economic development [2].

The formation of soil salinization is a complex ecological and environmental problem including various natural and anthropogenic factors. It is difficult to use single one factor to trace out the formation of soil salinization. The AHP could decompose complicated problems into hierarchies and combines quantitative analysis with qualitative analysis. AHP has been widely applied in the environment assessment. Tang establish the river health assessment model based on AHP [3], Zhou used AHP analyze the cause of alpine grassland degradation [4]. In this study, AHP method is employed to analyze the causes of soil salinization and efficiency of various restoring measures oil salinization in the Northern Yinchuan Plain.

\section{Method}

Study area. The study area is located in the upper reaches of the Yellow River in the northern Yinchuan plain. It ranges within latitudes $38^{\circ} 26^{\prime} 60^{\prime \prime}-39^{\circ} 14^{\prime} 09^{\prime \prime} \mathrm{N}$ and longitudes $105^{\circ} 57^{\prime} 40^{\prime \prime}-106^{\circ} 52^{\prime} 52^{\prime \prime}$ E. The mean annual precipitation for the period of $1990-2000$ is only $180.13 \mathrm{~mm}$, but evaporation reaches $1791.76 \mathrm{~mm}$, which is in agreement with semi-arid climate (Fig.1).

The study area is a traditional agricultural region, and the Yellow River is the main source for irrigation. Due to natural and human activities [5], the study area is under threat of soil salinization. The saline land was about $75.3 \%$ of the total area, the slight, moderate and severe salinization area 
accounting for $30.7 \% 、 24.1 \%$ and $20.5 \%$ respectively [6]. The soil salinization has been restricting the Northern Yinchuan Plain in social and economic development.

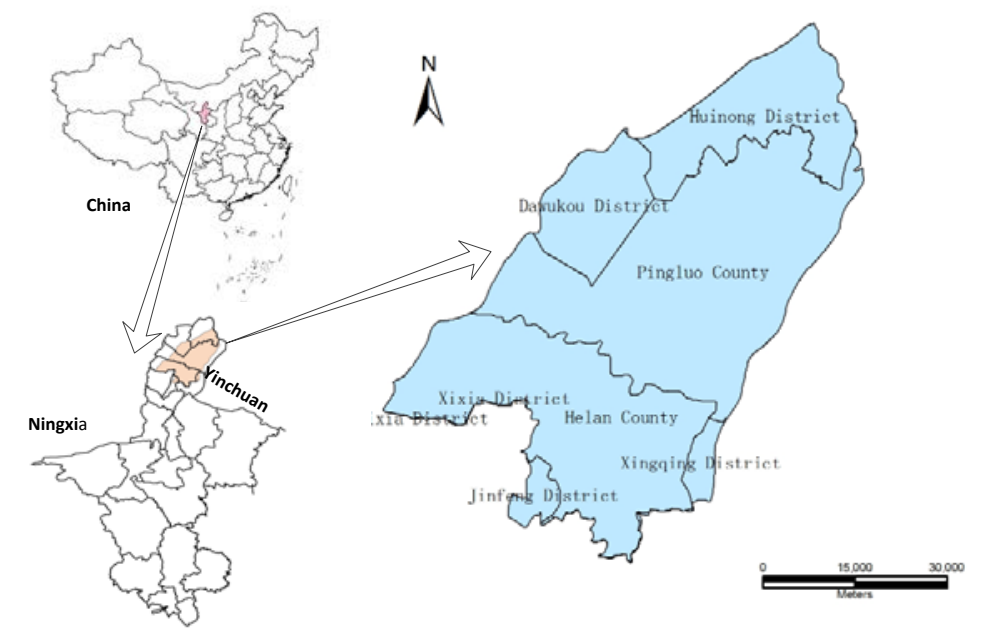

Fig.1 Location of the study area

Method. In this study, AHP was employed to analyze the causes of salinization and assess the efficiency of the measures on preventing soil salinization. AHP is a multicriteria decision making procedure for representing the elements of a problem hierarchically and ranking a set of alternatives or selecting the optimal alternatives by means of a series of pair-wise comparisons [7].

Step 1Structure a hierarchical model and identify the indicators. There are three layers included in the hierarchical model: the topmost layer is the goal of prevention and improvement of salinization, the second layer is the causes leading to salinization; the last layer is the corresponding measures (Fig.2).

According to numerous achievements on soil salinization in Northern Yinchuan Plain and semi-arid region [2, 5, 8], generally, semi-arid climate (F1) is a natural force inducing soil salinization; The parent material of soil (F2) provides salt resources for salinization; terrain and geomorphology (F3) have significant influence on the distribution of salinization; shallow groundwater table depth (F4) is main factor of soil salinization in the study area. In addition, groundwater with high salinity (F5) could accelerate salinization under intense evaporation. Furthermore, there are also many anthropogenic factors, which include unreasonable irrigation methods (F6), the poor drainage system (F7) and irrational cultivated practices (F8). According to the causes above, the measures for preventing salinization are summarized in the present study (Fig.2).

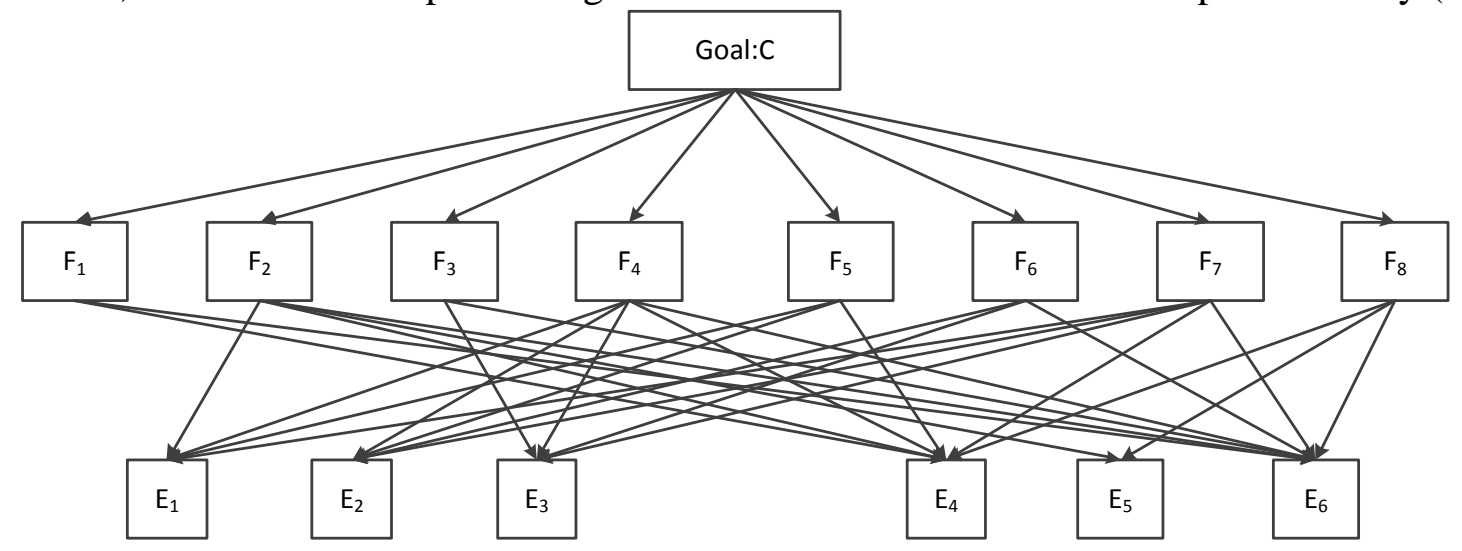

Fig.2 Structure of hierarchical model

Step 2 Construct pair-wise comparison matrix and weight the indicators. Based on the analysis of the causes above, the pair-wise comparison matrix combining 9-point scale developed by Saaty could be constructed. The matrix and the weights of second layer are shown in Table 1. 
Step 3Check consistency of evaluation. To verify the judgments expressed by the decision maker, Saaty has proposed the following consistency ratio (CR) [9].The value is a measure of how much variation is allowed and must be less than 0.1 :

Table 1 the matrix and weights for second layer

\begin{tabular}{c|ccccccccc}
\hline Causes & F1 & F2 & F3 & F4 & F5 & F6 & F7 & F8 & Weight \\
\hline F1 & 1 & 5 & 9 & 5 & 3 & 7 & 7 & 9 & 0.4073 \\
\hline F2 & $1 / 5$ & 1 & 7 & 3 & 3 & 5 & 5 & 7 & 0.2139 \\
\hline F3 & $1 / 9$ & $1 / 7$ & 1 & $1 / 5$ & $1 / 5$ & $1 / 3$ & $1 / 2$ & $1 / 2$ & 0.0231 \\
\hline F4 & $1 / 5$ & $1 / 3$ & 5 & 1 & $1 / 2$ & 2 & 2 & 5 & 0.0896 \\
\hline F5 & $1 / 3$ & $1 / 3$ & 5 & 2 & 1 & 5 & 5 & 3 & 0.1410 \\
\hline F6 & $1 / 7$ & $1 / 5$ & 3 & $1 / 2$ & $1 / 5$ & 1 & 2 & 3 & 0.0547 \\
\hline F7 & $1 / 7$ & $1 / 5$ & 2 & $1 / 2$ & $1 / 5$ & $1 / 2$ & 1 & 2 & 0.0406 \\
\hline F8 & $1 / 9$ & $1 / 7$ & 2 & $1 / 5$ & $1 / 3$ & $1 / 3$ & $1 / 2$ & 1 & 0.0298 \\
\hline
\end{tabular}

Consistency check: $\lambda_{\max }=8.5720, \mathrm{CI}=0.0817, \mathrm{RI}=1.41, \mathrm{CR}=0.058<0.1$

Step 4 Hierarchy general ranking. The general ranking is concluded by the elevated value of single indicator multiply its weight.

\section{Results and Discussion}

According to the procedure above, the total arrangement of hierarchy and weight of third layer are shown in Table 3 and the most effective measures of soil salinization will be realized.

Table 3Hierarchy general ranking of indicators of third layer

\begin{tabular}{cccccc}
\hline E1 & E2 & E3 & E4 & E5 & E6 \\
\hline 0.2995 & 0.0830 & 0.0487 & 0.3845 & 0.0136 & 0.1708 \\
\hline
\end{tabular}

The semi-arid climate (F1) and parent material of soil (F2) are considered as the main objective bases for soil salinization, which account for $40.7 \%$ and $21.4 \%$ respectively. Groundwater with high salinity (F5) and shallow groundwater table depth (F4) are the main factors influencing soil salinization holding $14.1 \%$ and 9\% respectively. Moreover, unreasonable irrigation methods (F6), the poor drainage system (F7) and irrational cultivated practices (F8) are factors accelerating soil salinization (Fig.3).
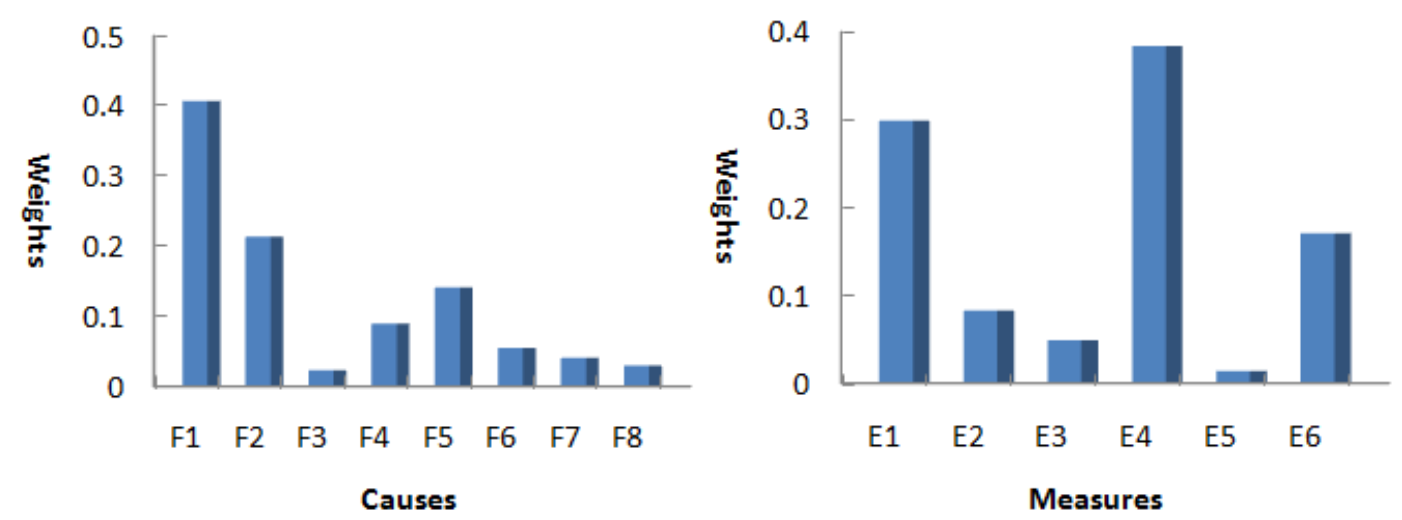

Fig.3 The distribution of integrated weights of various causes and restoring measures

The comparison of the efficiency of the various measures is shown in Fig.2. The measures of afforestation and planting salt-tolerant plants (E4) and improving drainage system (E1) are essential for preventing soil salinization, which occupies $38.4 \%$ and $29.9 \%$ respectively. Adoption of reasonable planting patterns (E6) and controlling seepage from canal (E2) are also effective, which possess $17 \%$ and $8.3 \%$ respectively. Additionally, land leveling (E3) and Chemical improvement (E5) are also playing irreplaceable role on soil salinization prevention. 
Many experts agreed that the shallow groundwater table depth is the crucial factor influencing soil salinization. However, improving drainage system (E1) is not the most effective measure according to Fig.2. It could be interpreted by the measure of planting salt-tolerant plants (E4) and controlling seepage from canal (E2) can decline the groundwater level effectively. Therefore, the weight of improving drainage system (E1) is not the largest. The results indicate that soil salinization of the Northern Yinchuan Plain will be improved by taking comprehensive measures and the water-salts balance will be realized ultimately.

\section{Conclusion}

In this study, AHP technique is employed to assess the efficiency of various measures on preventing soil salinization in the Northern Yinchuan Plain. The method is comparatively objective and comprehensive. Furthermore, the application of AHP on the soil salinization in the study area could transform the qualitative indicator (such as the causes and measures of salinization) into quantitative indicator. According to results, afforestation and planting salt-tolerant plants and improving drainage system were better than other measures on preventing soil salinization. Therefore, the calculated results can provide basis and reference for preventing soil salinization.

\section{Acknowledgements}

The research was supported by the National Natural Science Foundation of China (41172212).

\section{References}

[1] Y.G.Wang, D.N.Xiao, Y.Li, X.Y.Li, Soil salinity evolution and its relationship with dynamics of groundwater in the oasis of inland river basins: case study from the Fubei region of Xinjiang Province China, J. Environmental Monitoring and Assessment. 140(2008) 291-302.

[2] X.Xu, G.H.Huang, C.Sun, Assessing the effects of water table depth on water use, soil salinity and wheat yield: Searching for a target depth for irrigated areas in the upper Yellow River basin, J. Sci. Agriculture Water Management. 125(2013)46-60.

[3] X.Q.Tang, Y.Xie, Analysis Hierarchy Process Model for Plain River Reach Health Assessment of the Luanhe River, J. Wuhan University Journal of Natural Sciences. 14(2009)537-542.

[4] H.K.Zhou, X.Q.Zhao, L.Zhou, Application of Analytic Hierarchy Process on the Alpine Grassland Degradation in the Source Region of the Yangtze AND Yellow Rivers, J. Resources Science. 27(2005)(in Chinese)

[5] J.H.Wu, P.Y.Li, H.Qian, Assessment of soil salinization based on a low-cost method and its influencing factors in a semi-arid agricultural area, northwest China, J. Sci. Environ Earth. 71(2014)3465-3475.

[6] Y.Fang, Study on Soil Salinization in Typical Areas of North Central Ningxia Based on WET, D. (2012)(in Chinese)

[7] M.H.Ravesh, H.Ahmadi, G.Zehtabian, Application of sensitivity analysis for assessment of de-desertification alternatives in the central Iran by using Triantaphyllou method, J. Environ MonitAssess. 179(2011)31-46.

[8] X.Xu, Huang, G.H.Huang, Z.Y.Qu, L.S.Pereira, Using MODFLOW and GIS to Assess Changes in Groundwater Dynamics in Response to Water Saving Measures in Irrigation Districts of the Upper Yellow River Basin, J. Water Resour Manage. 25(2011)2035-2059.

[9] G.B.Kim, J.S.Ahn, A.Marui, Analytic hierarchy models for regional groundwater monitoring well allocation in Southeast Asian countries and South Korea, J. Sci. Environ Earth. 59(2009)325-338. 